\title{
Interventional Radiology Robot for CT and MRI Guided Percutaneous Interventions
}

\author{
Nikolai Hungr ${ }^{1}$, Céline Fouard ${ }^{1}$, Adeline Robert ${ }^{1}$, \\ Ivan Bricault ${ }^{2}$, and Philippe Cinquin ${ }^{1}$ \\ ${ }^{1}$ UJF-Grenoble 1 / CNRS / TIMC-IMAG UMR 5525 (GMCAO), Grenoble, F-38041, France \\ \{nikolai.hungr, celine.fouard\}@imag.fr \\ ${ }^{2}$ Service Central de Radiologie et Imagerie Médicale, CHU Grenoble, \\ 38700 La Tronche, France
}

\begin{abstract}
This paper introduces a new patient-mounted CT and MRI guided interventional radiology robot for percutaneous needle interventions. The 5 DOF robot uses ultrasonic motors and pneumatics to position the needle and then insert it progressively. The needle position and inclination can be registered in the images using two strategically placed fiducials visible in both imaging modalities. A first prototype is presented and described in terms of its sterilization, CT and MRI compatibility, and precision. Tests showed that 1) it is entirely sterilizable with hydrogen peroxide gas, 2) no image artifacts or deformations are noticeable in the CT and MRI images, 3) does not affect the SNR of MR images, and 4) its mechanical error is less than $5 \mathrm{~mm}$.
\end{abstract}

Keywords: Interventional radiology, Robotics, CT, MRI, Image guided, Ultrasonic motors, Magnetic resonance compatibility, Sterilization.

\section{Introduction}

Percutaneous interventional radiology (IR) is a field that consists of diagnostic and therapeutic procedures such as image-guided biopsies, cancer treatment by radiofrequency ablation, cryoablation and high-intensity focused ultrasound (HIFU), abscess and pleural drainage, and vertebroplasty. In 2008, 11.5 million IR acts were realized in the US, of which $35 \%$ where non-cardiovascular. The popularity of these procedures has incited a growing interest in robotic-assisted techniques.

Of the primary imaging techniques used in IR, CT and MRI are of particular interest due to their ability to view any part of the body in three-dimensions and with typically higher definition. Both modalities have their advantages. CT images have high resolution whereas MRI is non-irradiating, has better contrast density for soft tissues, and allows for thermal imaging for cryoablation for example, as well as noninvasive real time imaging that introduces the possibility of motion tracking and compensation. These differences make CT and MRI imaging complementary, making IR interesting in both modalities.

CT-guidance is a well established technique for percutaneous needle interventions. Some of the main difficulties encountered in conventional CT-guided IR are accessibility due to constrained tunnel dimensions, lengthy and iterative steps that 
affect patient comfort, difficulty for the clinician to visualize the needle trajectory on the patient, as image planning is done in the distant control room, and important complications when inclining the needle in two directions. MRI-guidance, on the other hand, remains uncommon in clinical practice due to cost, tunnel size and material compatibility constraints.

In this paper, we present a new, patient-mounted multimodal, MRI and CT compatible interventional radiology robot based on our previously published LPR robot [1]. The primary goal of the robot is to increase the scope of percutaneous interventions available to clinicians by 1) increasing needle insertion accuracy by allowing accurate location of needle position and inclination in the images combined with progressive needle insertion, 2) allowing more complex 3DOF approach angles (between the ribs or deep insertions, for example), 3) reducing the number of image acquisitions required (and hence decreasing radiation doses in CT), 4) improving intervention efficiency, and 5) offering the possibility of MR-guided interventions (for example for patients with certain contraindications to CT contrast agents, or for better visualization of equal-density tumors, etc.).

\section{State of the Art}

Most of the difficulties described in the previous paragraph could be addressed through robotic assistance, and indeed, a multitude of systems have been described in the literature. These systems can be classified into two categories: bed-mounted robots and patient-mounted robots. An example of the first class of robots is the AcuBot [2][3], a CT guided IR robot, which uses a bridge-like macro-micro architecture to insert a needle with $1-2 \mathrm{~mm}$ precision. Another similarly bridgemounted robot is the MRI-guided Innomotion system, which uses pneumatic actuators coupled with optical sensors to drive the robot, while needle insertion remains manual, with sub-millimetric accuracy [4]. A third example is the B-RobII system which uses two parallel fingers to align the needle above a target, allowing the clinician to insert the needle manually, with a precision of $0.88 \mathrm{~mm}$ [5].

Patient-mounted systems have the difference of being smaller and able to move with the patient. The effect of motion due to patient discomfort as well as the motion of the insertion point on the patient's skin during breathing can thus be minimized to a certain extent. The CT-Bot is one such robot [6] that uses ultrasonic motors to power a parallel-structure with 5 degrees of freedom (DOF). It uses a single line-fiducial mounted on the base of the robot for registration with the CT images, allowing an accuracy of $<5 \mathrm{~mm}$ at typical needle insertion depths. The Robopsy is another example of such a CT-guided robot that has 3 DOF for needle orientation and insertion [7], while positioning at the skin insertion point is manual. Neither of these robots are MRI compatible.

The systems described above show the important potential benefit that roboticassistance can have for improving the quality and feasibility of percutaneous IR procedures. The Light Puncture Robot (LPR) [1] developed in our laboratory, consisting of a patient-mounted needle insertion module stretched between four straps can be pulled individually to control the needle position. The major contribution of this project compared to other existing systems is its compatibility with both CT and MRI guided percutaneous procedures, significantly enlarging its clinical scope. 

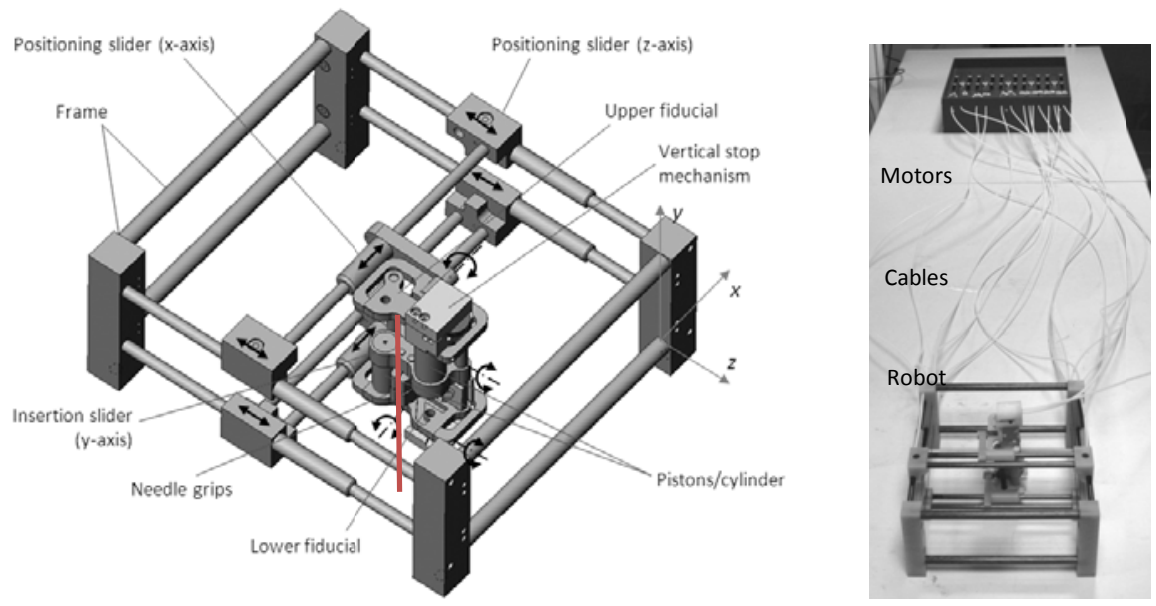

Fig. 1. New robot architecture. The needle is denoted by a vertical red line in the left image.
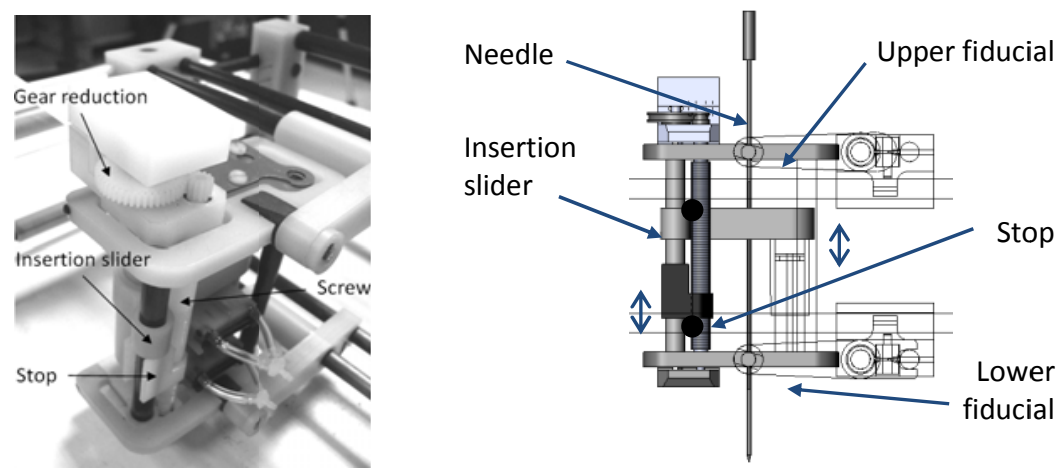

Fig. 2. Stop mechanism for setting the insertion stroke

Although the animal testing reported in [1] showed the feasibility and accuracy of the LPR in a CT-guided environment, a few limitations were revealed to the design. Firstly, although the needle insertion module was light and relatively compact, the size of the surrounding frame limited the size of patient that could fit inside. Additionally, the pneumatic actuators resulted in slow motions and significant noise during pressure-release.

This paper introduces the new LPR design, along with some preliminary precision and compatibility results, and sterilization tests as we prepare for phantom and mock patient trials.

\section{Robot Description}

The robot architecture is shown in Figure 1. It is based on a 5 DOF parallelogram design, in which the needle is held by two parallel platforms, $85 \mathrm{~mm}$ apart, that enable 
a maximum planar translation of $135 \mathrm{~mm}$ and a maximum needle inclination of $\pm 60^{\circ}$ in one direction and $\pm 15^{\circ}$ in the other. The needle is held by two independent grippers the upper one being mounted on a vertical slider and providing the needle insertion force, as shown in Figure 2. The frame is strapped to the patient's body to follow external movements.

Needle insertion is done incrementally by sequentially activating the two needle grippers and the insertion slider (Figure 2). The stroke distance (adjustable between 0 and $40 \mathrm{~mm}$ ) is regulated by a controllable stop that blocks the vertical slider's path. The stop height is regulated by a screw connected to a set of reduction gears.

Translation of the four $\mathrm{x}$ and $\mathrm{z}$ sliders is achieved through four Shinsei USR60E3N ultrasonic motors. Their torque $(\max .1 \mathrm{Nm}$ ) and speed (rated 100rpm) ratings are easily sufficient for the low friction and low reduction characteristics of the robot. As studies have shown that these motors are capable of affecting an MRI image when powered inside the tunnel [8], it was decided to house them in a separate container placed on the scanner or MRI bed, at the patient's feet (see Figure 1, right). They are connected to the robot by $1.5 \mathrm{~m}$ long cables and housing, ensuring that they are never inside the tunnel. Each motor activates a pair of rack and pinions, which in turn pull the cables to translate the respective robot slider. The cable housing is made of Teflon, to reduce cable friction, while the cables are made of low stretch $0.4 \mathrm{~mm}$ diameter Spectra thread, typically used in archery. Separating the motors from the robot also reduces the robot's weight on the patient's body.

While the insertion stop mechanism is controlled by an ultrasonic motor, the insertion and retraction of the insertion slider as well as the two needle grippers are powered by pneumatics. This was chosen in order to give sufficient power and speed to these vital elements of the robot. At a typical hospital air pressure of 4 bars, the needle insertion force is $>50 \mathrm{~N}$, while the gripping force is $8 \mathrm{~N}$. The latter increases to $13 \mathrm{~N}$ at 5 bars, which is largely enough for typical needle insertion forces. Air pressure can be provided either by the hospital air supply that is usually found in or near the imaging rooms, by bottled medical air, or by a small electrical pump, the latter two being, of course, located outside the imaging room, particularly in the case of MRI.

All the robot and motor unit materials consist of a combination of CT and MRIcompatible delrin, epoxy resin, carbon fiber and nylon materials. Fiducials, mounted on both the parallel platforms and both visible in CT and MRI, allow for direct tracking of the needle position and inclination, instead of just the robot frame, as is the case in other robots. This increases accuracy and reliability.

The robot control box, located outside the imaging room, houses the motor controllers and pneumatic solenoid valves. The electronic cables and air hoses pass through the service hatch of the scanner/MRI room. The PC used to control the robot is connected to the hospital network, allowing it to obtain the images coming from the imager.

\section{Robot Evaluation}

\subsection{Sterilization}

Along with the functional aspects of the design, sterilization is a vital element to consider. Although not yet fully incorporated into this first prototype, a study of its 
sterilizability has been carried out. Percutaneous IR needle interventions can be considered low risk procedures, as they occur in the open environment of the scanner and MRI rooms, and as the inherent risk of a single needle insertion is relatively small. The robot's primary condition for sterilization must be that there be no risk of contact between a non-sterile component of the robot and the needle or the clinician.

Since the needle is located within the confines of the robot's frame, requiring the clinician to reach inside to handle the needle, and since there are many moving elements that would be difficult to simply cover with sterile covers or drapes, it was decided to sterilize the entire robot and part of the cables leading to the motor unit. Any potential air leaks coming from the pneumatic system were deemed irrelevant, as the air in the imaging room is untreated, ambient air in any case.

Due to the numerous moving parts, with 14 cables running in and out of their $1.5 \mathrm{~mm}$ inner diameter cable housings, and numerous heat sensible materials, sterilization by hydrogen peroxide gas (such as the STERRAD® system, from Advanced Sterilization Products) was chosen. Three aspects for successful sterilization were explored.

First, to fit into the smallest typical sterilization chamber $(170 \times 300 \times 600 \mathrm{~mm})$ it is necessary to disconnect the robot from the motor unit. Although not yet incorporated into the prototype described here, a cable connector has been design. This will allow the entire robot and nearby cables to be placed in the sterilization chamber at once.

Second, as the compatibility of carbon fiber with hydrogen peroxide gas was unkown, we decided to test this compatibility. We passed two carbon fiber samples through 25 normal sterilization cycles using the STERRAD® NX machine available to us in our partner hospital (cycle time $=38 \mathrm{~min}$; temperature $=45^{\circ} \mathrm{C}$; pressure $=$ 1.013 bar). Visual microscopic comparison of the surfaces of these samples with nonsterilized samples, showed no evidence of surface decomposition, microfractures, or delamination of the fibers. Bend to fracture tests on these sample bars also showed no noticeable change in fracture strength and fracture mode. As the robot frame undergoes minimal loads during operation, these results were deemed satisfactory.

Third, as temperature is not an acting sterilization agent in the sterilization technique chosen, it is necessary that all parts of the robot be reachable by the gas. This is especially the case for the cables hidden inside the cable housing, which during robot operation will emerge within close range of the needle, as they are pulled in and out. To verify the successful sterilization inside the cable housing, an experiment was undertaken in which 4 Spectra cables were impregnated with Geobacillus Stearothermophilus spores (standard bacterium used for validation of sterilization studies, including autoclave and gas-based techniques) and inserted into $30 \mathrm{~cm}$ lengths of cable housing. Three of these were passed through a single STERRAD® sterilization cycle, along with a batch of control spores in an open Eppendorf tube. The three sterilized samples, the control spores and the one non-sterilized sample, were then put into cultivation at $60^{\circ} \mathrm{C}$, and the optical densities (OD) were measured using a spectrometer for each cultivation solution at $24 \mathrm{~h}$ and $72 \mathrm{~h}$. At both instances, only the non-sterilized sample showed any sign of bacterial proliferation $(\mathrm{OD}=0.952$, compared to $\mathrm{OD}<0.014$ for sterilized samples), confirming that the cables were successfully sterilized. As the cable housing will be disconnected from the robot during sterilization, this experiment was deemed appropriate and conclusive. 

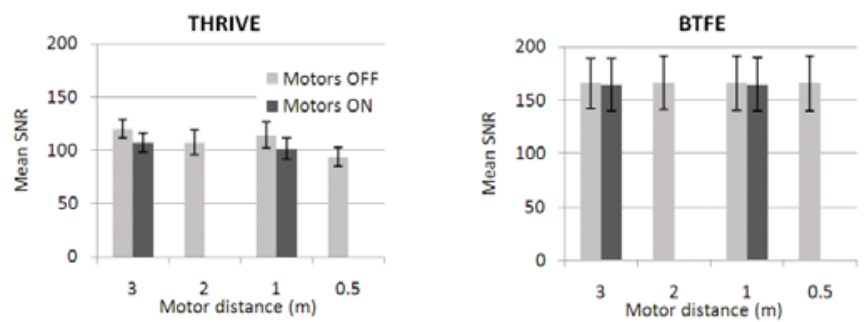

Fig. 3. Average SNR for each set of THRIVE and BTFE images taken during MRI compatibility tests. Motor distance was measured from the opening of the MRI tunnel.

\subsection{CT and MRI Compatibility}

To verify the CT, and in particular MRI compatibility of our prototype, we conducted three experiments. In the first test, we imaged the robot in a Siemens Somatom Sensation 16 CT scanner at a resolution of $512 \times 512$ and a slice thickness of $1 \mathrm{~mm}$. The images showed no signs of streaking or beam hardening, artifacts that could affect the clinical image quality. Also, compared to the previous LPR design, this robot stands on four legs, bringing the bulk of the robot away from the patient's skin, making it easily distinguishable in the CT images.

In the second test, we measured the signal to noise ratio (SNR) in a series of MRI image volumes, with the robot in place and with the motor unit at increasing proximity to the MRI tunnel $(3 \mathrm{~m}, 2 \mathrm{~m}, 1 \mathrm{~m}$, and $0.5 \mathrm{~m})$. The SNR was measured in exactly the same manner as described in [1] and [8], using a phantom bottle in a Philips Achieva 3.0T MRI scanner. BTFE ${ }^{1}$ and THRIVE ${ }^{2}$ sequences, typically used during IR, were taken. The results of the various measurements made are shown in Figure 3 and are within the ranges reported in [1]. We note that all signal to noise ratios are comparable and acceptable.

In the third test, using the same images as in the second test, the upper and lower robot fiducials were segmented using identical thresholds for all the image volumes. Two measurements were made and are summarized in Table 1: the average variation (expressed as a standard deviation) of the center of motion (COM) for each segmented image and the average volume of segmented pixels for all segmented fiducials. The variations of both measurements did not show any sort of pattern based on the motor distance or state. Note that in this test, the registration of the segmented image COM to the true physical robot COM was not considered, as our primary interest here was the effect of the robot and motors on image quality.

Although the two MRI tests presented are preliminary and will have to be furthered on actual patients, it is important to note that 1) the motors will never be actuated during image acquisition, and 2) the motors will never be inside the imaging tunnel. The results were therefore very encouraging.

${ }^{1}$ Balanced Turbo Field Echo: Slice thickness $=5 \mathrm{~mm}$, resolution $=256 \times 256$, slices $=15$, slice spacing $=6.5 \mathrm{~mm}, \mathrm{TR}=2.62 \mathrm{~ms}, \mathrm{TE}=1.31 \mathrm{~ms}$, flip angle $=45^{\circ}$.

2 T1 Weighted High Resolution Isotropic Volume Exam: Slice thickness $=4 \mathrm{~mm}$, resolution = $384 \times 384$, slices $=80$, slice spacing $=2 \mathrm{~mm}, \mathrm{TR}=3.03 \mathrm{~ms}, \mathrm{TE}=1.42 \mathrm{~ms}$, flip angle $=10^{\circ}$. 
Table 1. Average variations (expressed as standard deviation) of the segmented upper and lower fiducials for motor distances of $0.5 \mathrm{~m}$ (motors OFF), $1 \mathrm{~m}$ (OFF and ON), $2 \mathrm{~m}$ (OFF) and $3 \mathrm{~m}(\mathrm{OFF}$ and $\mathrm{ON})$ from the MRI tunnel opening. Note: $\mathrm{ON}=$ in motion (i.e. worst case).

\begin{tabular}{l|cc|cc}
\hline & \multicolumn{2}{|c|}{ Average variation of pixel volume } & \multicolumn{2}{|c}{ Average variation of COM (mm) } \\
Fiducial: & Upper & Lower & Upper & Lower \\
\hline BTFE & $8 \%$ & $7 \%$ & 0.75 & 0.30 \\
THRIVE & $15 \%$ & $15 \%$ & 1.02 & 0.47 \\
\hline
\end{tabular}

\subsection{Robot Accuracy}

A preliminary test has been run on the robot to determine its needle positioning error before advancing to more in depth needle insertion experiments on phantoms and mock patient trials. The test involved moving the robot inside an MRI imager and comparing images taken before and after the motion. By segmenting the fiducials in both volumes, the distance moved by the robot can be compared to the commanded distance. This comparison gave us an idea of the total error, including robot motion and imaging errors. Segmentation of the images was done manually, using smoothing, thresholding and basic binary processing to isolate the fiducials and then to calculate their centers of mass (COM) within each image. The latter was used to calculate the distance moved. From a total of six repeated $50 \mathrm{~mm} \times$ and $y$ translations, the resulting error was found to be $4 \mathrm{~mm}(\mathrm{SDev}=1.1)$ for the upper fiducial and $5 \mathrm{~mm}(\mathrm{SDev}=1.2)$ for the lower fiducial.

These errors come from two sources. First, the images used in this analysis were of fairly low resolution (384 x 384 pixels, 100 slices, $0.9766 \mathrm{~mm}$ pixel size, $2 \mathrm{~mm}$ image spacing), significantly affecting the reported error. Second, the mechanical stretching of the cables during motion, result in some hysteresis between the motor movement and the respective slider movement $1.5 \mathrm{~m}$ away. This effect was minimized through material selection (use of stiff, low friction materials), but is impossible to eradicate completely. To decrease this error, we are looking, on one hand, into a better way of equalizing the cable tension throughout the robot, and on the other to develop an algorithm to predict the hysteresis.

\section{Conclusion}

We are continuing development on this prototype in order to carry out phantom needle insertion tests in both CT and MRI machines, as well as further mock patient tests in the MRI machine. Specific research aspects include the development of automatic fiducial segmentation algorithms, their incorporation into the robot kinematics, and ergonomic, size and actuation redundancy improvements (in particular, to increase the safety of the needle insertion module and to ensure no unwanted motion of the linear slides during needle insertion), robot-to-patient fixation design, and hysteresis tests on the cables, to prepare the robot for clinical trials.

Acknowledgments. We would like to acknowledge Nabil Zemiti for spearheading the original design of this robot, Jean Pierre Alcaraz for his expertise with the bacterial study and the departments of sterilization, security and hygiene of the Grenoble University Hospital. 


\section{References}

1. Zemiti, N., Bricault, I., Fouard, C., Sanchez, B., Cinquin, P.: LPR: A CT and MRCompatible Puncture Robot to Enhance Accuracy and Safety of Image-Guided Interventions. IEEE/ASME Transactions on Mechatronics 13(3), 306-315 (2008)

2. Stoianovici, D., Cleary, K., Patriciu, A., Mazilu, D., Stanimir, A., Craciunoiu, N., Watson, V., Kavoussi, L.: AcuBot: a robot for radiological interventions. IEEE Transactions on Robotics and Automation 19(5), 927-930 (2003)

3. Cleary, K., Watson, V., Lindisch, D., Taylor, R.H., Fichtinger, G., Xu, S., White, C.S., Donlon, J., Taylor, M., Patriciu, A., Mazilu, D., Stoianovici, D.: Precision placement of instruments for minimally invasive procedures using a "needle driver" robot. Int. J. Med. Robot. 1(2), 40-47 (2005)

4. Melzer, A., Gutmann, B., Remmele, T., Wolf, R., Lukoscheck, A., Bock, M., Bardenheuer, H., Fischer, H.: INNOMOTION for percutaneous image-guided interventions: principles and evaluation of this MR- and CT-compatible robotic system. IEEE EMB Magazine 27(3), 66-73 (2008)

5. Kronreif, G., Fürst, M., Ptacek, W., Kornfeld, M., Kettenbach, J.: Robotic System for Image Guided Therapie - B-RobII. In: RAAD Workshop, BFD-022 (2006)

6. Maurin, B., Bayle, B., Piccin, O., Gangloff, J., de Mathelin, M., Doignon, C., Zanne, P., Gangi, A.: A Patient-Mounted Robotic Platform for CT-Scan Guided Procedures. IEEE Transactions on Biomedical Engineering 55(10), 2417-2425 (2008)

7. Walsh, C., Hanumara, N.C., Slocum, A.H., Shepard, J.A., Gupta, R.: A Patient-Mounted, Telerobotic Tool for CT-Guided Percutaneous Interventions. J. of Med. Devices 2(1), 011007.1-011007.10 (2008)

8. Fischer, G.S., Krieger, A., Iordachita, I.I., Csoma, C., Whitcomb, L.L., Fichtinger, G.: MRI Compatibility of Robot Actuation Techniques - A Comparative Study. In: Metaxas, D., Axel, L., Fichtinger, G., Székely, G. (eds.) MICCAI 2008, Part II. LNCS, vol. 5242, pp. 509-517. Springer, Heidelberg (2008) 